\title{
Temperature Level Fiber Sensor Network
}

\author{
J.M. López-Higuera, L. Rodriguez-Cobo, Jesús Castrellón Uribe, A. Quintela and M. Lomer \\ Photonics Engineering Group, University of Cantabria, $R \& D$ Building, 39005, Santander, Spain \\ lopezhjm@unican.es
}

\begin{abstract}
A temperature level fiber sensor network is proposed and demonstrated. Each inline transducer is based on a FBG-SMA wire structure working as an on/off optical device being interrogated using a time domain technique.

OCIS codes: (060.3735) Fiber Bragg gratings; (280.4788) Optical sensing and sensors
\end{abstract}

\section{Introduction}

Fiber Bragg Grating (FBG) technology have been widely investigated as basic structure of fiber telecommunication devices [1], lasers [2], as the basic structure of optical sensors [3, 4,] and sensor networks [5]. However, the development and availability of new materials enables the conception of new fiber devices using their technical properties in conjunction with some current fiber optic structures. Very recently a new fiber transducer based on a Shape Memory Alloy (SMA) wire and the FBG technologies are properly combined to take the advantages of both of them and to enable the creation of a new on/off optical fiber device (TelMA) able to be used in sensing field [6]. Based in this new device a new fiber optic sensor network (TeLMAN) is proposed and demonstrated. The principle, and preliminary results are presented and discussed in this paper.

\section{Principle and demonstration}

The TeLMA fiber transducer can be used to detect a maximum temperature level, TL, and can be used both as a point or quasi-distributed fiber sensors systems. The TeLMA transducer structure is constituted by a Fiber Bragg Grating (FBG) properly fixed to a SMA preloaded with a Bias Force (FB). The Bias Force provokes on the SMA wire length, 1, a wire elongation $\square$. When the transducer temperature is over a given temperature level (TL) the wire recovers its original length producing a contraction on the FBG structure length $(\Delta \mathrm{l})$ and, hence a substantial reduction on its Bragg wavelength spectral response due to the applied strain (- $\Delta \mathrm{l} / \mathrm{l})$. The TeLMA working principle is illustrated in Fig. 1.
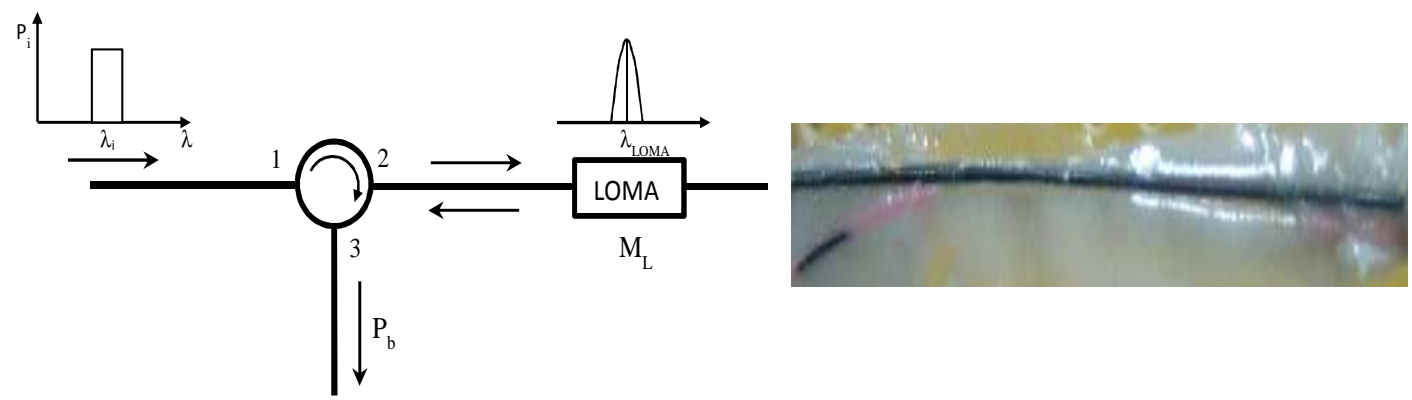

Figure 1. Basic fiber sensor concept architecture. $M=$ measurand (in this case is $T$ ); $M_{L}=$ measurand level; $P_{i}$ and $P_{b}$ sensor input and output (back) optical Powers; $\lambda_{i}, \lambda_{\text {TeLMA }}$ and $\lambda_{b}$ are the input, TelMA and back optical wavelength of the signal spectra respectively. The inset is a photo of the key detail of each transducer: a FBG glued to a SMA pre-loaded wire. For T > TL, the $\lambda_{\text {TeLmA }}$ is decreased and is then insider the pulse sprectrum and then an optical power $(\mathrm{Pb})$ appears at the output of the TeLMA transducer; for T< TL, then $\lambda_{\text {TeLMA }}$ is out of the pulse sprectrum and hence the optical power $(\mathrm{Pb}=0)$ at the output of the TeLMA transducer.

When the TeLMA fiber structure is optically interrogated, then an on/off optical sensor device can be constructed (figure 1). When the temperature level, $\mathrm{T}_{\mathrm{L}}$, is over passed, some optical reflected power $\left(\mathrm{P}_{\mathrm{b}}\right)$ is offered by the output sensor port (On) and when the temperature is below the mentioned level (threshold) no reflected optical power is offered by the output sensor port (Off). If the TeLMA structure is cooled to the original temperature then the SMA wire recover its original length, 1 , and its original FBG spectral response $\left(\lambda_{\text {LOMA }}\right)$ is also recovered too.

The experimental TelMA transducer dynamic response used in this paper is shown in the figure 2. From the later it can be deducted that a change of 1,6 $\mathrm{nm}$ in the device Bragg wavelength is obtained 


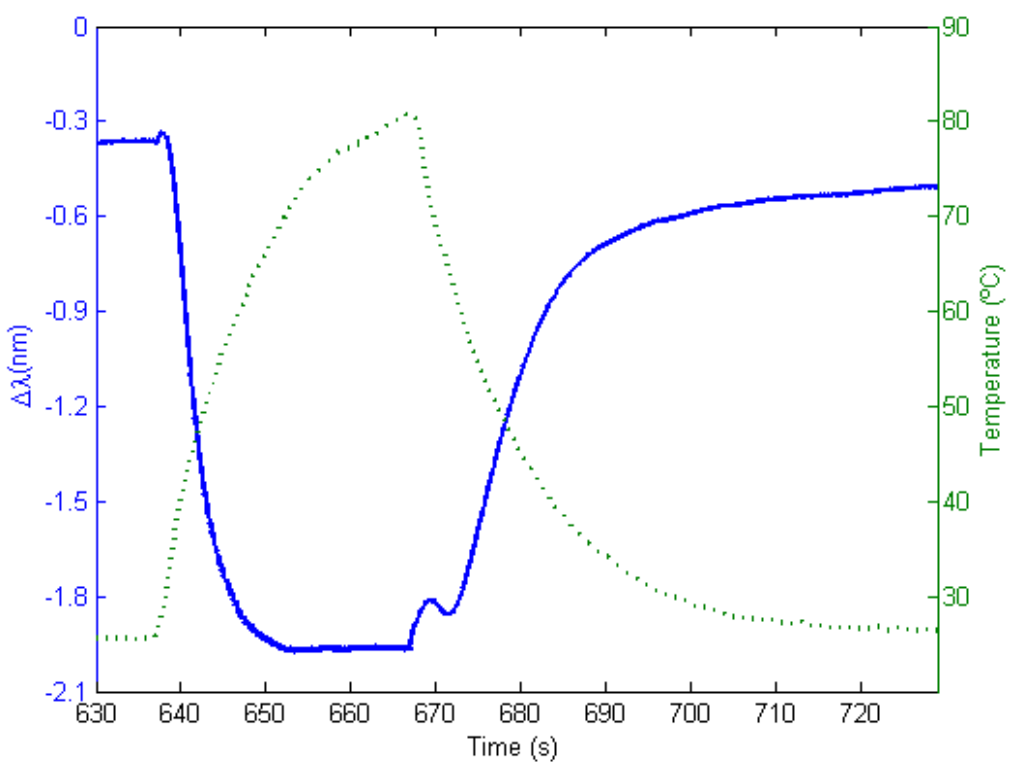

Figure 2. TeLMA wavelength change, $\lambda_{\text {TelmA }}$, evolution (solid blue line) for a heat pulse of $80^{\circ} \mathrm{C}$ peak (dotted green line) over the SMA wire temperature level $\left(\mathrm{T}_{\mathrm{L}}=70^{\circ} \mathrm{C}\right)$ of the device. The TeLMA temperature is measured in the laboratory using an infrared camera (FLIR ThermaCam SC2000).

It must be noticed that the Bragg wavelength response can by tuneable (in a given range) by different temperature levels by using different kind of SMA wires. It is also demonstrated that finished the heat pulse, the wavelength response is recovered following the cooling process of the TeLMA structure and according to the temperature-strain SMA wire behaviours.

As shown in Fig. 3, the sensor Network proposed in this paper is integrated by two main parts: the transducer (TeLMAN) and the optoelectronic or interrogation unit. The former, TeLMAN, consist in a high number of concatenated TeLMA transducers in which the fiber gratings are fabricated with low reflection rates, $\mathrm{R}_{\mathrm{n}}(1 \%$ to $5 \%)$ depending of the required number $(\mathrm{N})$ of quasi-distributed sensing points per channel. The higher the number $\mathrm{N}$ the lower the reflection rate value $\mathrm{R}_{\mathrm{n}}$.

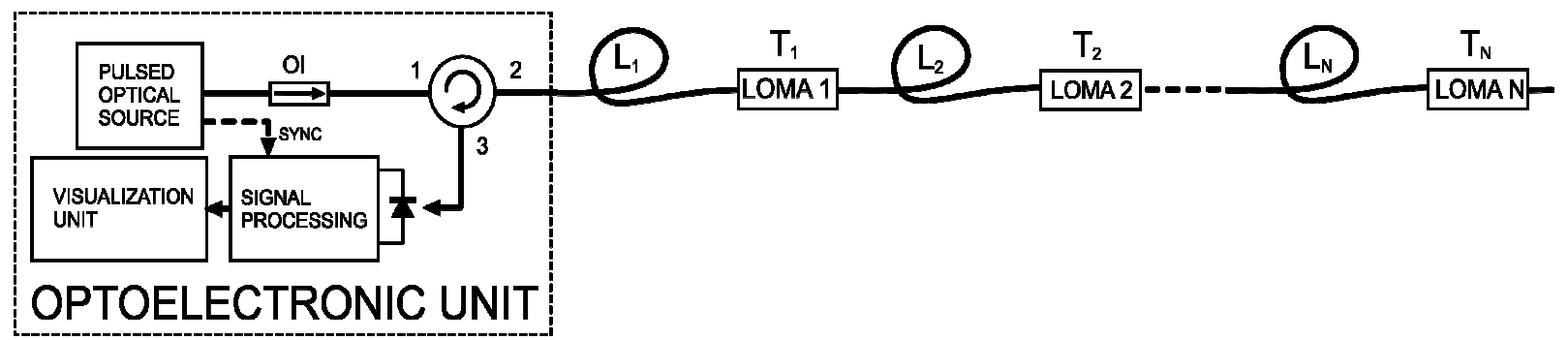

Figure 3. Illustration of the sensor networkr, TeLMAN set-up. Only one transducer channel is depicted.

The light from the pulsed laser is launched to the quasi-distributed transducer through an optical insulator and a circulator. The reflective response of each TeLMA transducer Network is then launched to a photo-detector from the port 3 of the optical circulator. Then the photo-detected signal is processed and properly represented on the visualization component. The photodetector is properly synchronized with each pulse produced by the laser what narrow line-width, Dl, is centred on the $l_{\text {laser }}$ wavelength. The latter, is properly selected to be placed on the $1 \mathrm{~T}_{\text {TelMA- }}$ ON (below 1 TeLMA-OFF) what means that when the temperature transducer is below the $\mathrm{T}_{\mathrm{L} n}$ (of the n-TelMA transducer) the device $n$ is optically transparent (only the connection losses are computed). However when the temperature on the transducer $n$ is higher than the $\mathrm{T}_{\mathrm{Ln}}$ then the $\mathrm{R} \%$ of the incoming optical power to the device $\mathrm{n}$ is returned to the photo-detector and then the un-desirable event $\left(\mathrm{T}_{n}>\mathrm{T}_{\mathrm{L} n}\right)$ is detected through the signal protcessing algorithm. As the signal processing unit (with its photo-detector) is syncronized with the each optical pulse 
produced by the laser, using an Optical Time Delay Reflectometric technique the position of each transducer is properly clearly obtained.

A picture of the representation of the data inside the visualization unit can be observed in the figure 4 . Each position device (time or fiber length from the optoelectronic unit) is known from their very small insertion losses or by their reflection optical power for TeLMA devices on OFF or ON state respectively.

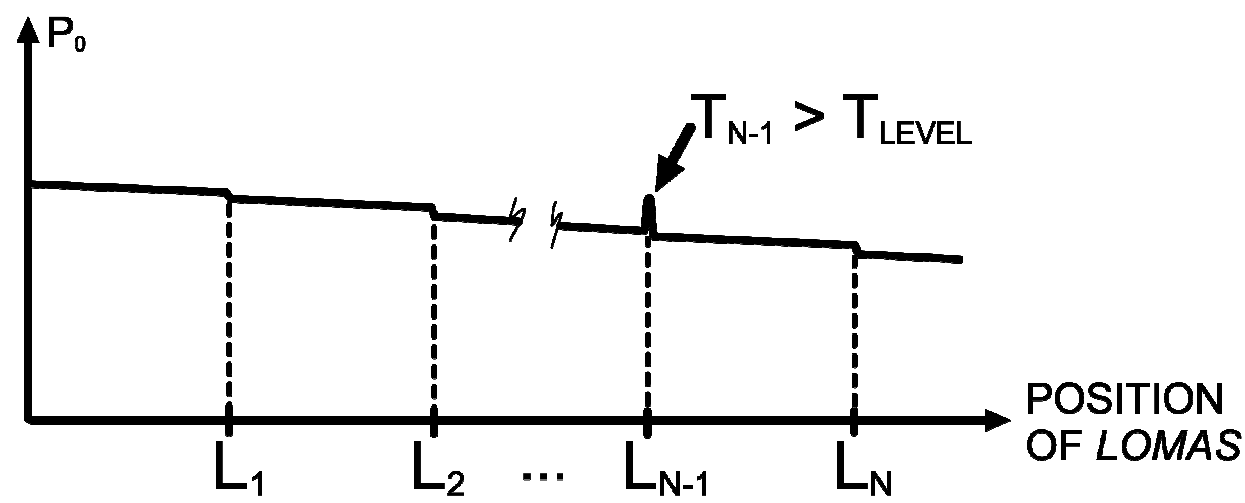

Figure 4. Visualization of the position of each sensing point in the TeLMAN sensor Network. The transducers N-1 is activated due to that its temperatures are higher than its admitted maximum level $\left(\mathrm{T}_{n}>\mathrm{T}_{\mathrm{L} n}\right)$.

\section{Conclusion}

In this paper, a temperature level fiber sensor network, TeLMAN, is proposed and successfully demonstrated. Each inline transducer, based on a FBG-SMA wire structure -working as an on/off optical-, is interrogated using an optical time domain reflectometric technique. Each in line time domain multiplexed TeLma transducer is based on a low reflection fiber grating and a shape memory alloy wire that works as an on/off optical device. The latter only offer output light when the structure temperature $(\mathrm{T})$ overpass a given temperature level $\left(\mathrm{T}>\mathrm{T}_{\mathrm{L}}\right)$ or temperature threshold that is established by the SMA wire behaviours. Several tens of sensing points can be concatenated in each line (channel) of the TeLMAN using low reflective FBG's in each TeLMA transducer. Several hundred or thousand sensing points can be deployed properly using and multichannel scheme.

This quasi-distributed temperature level sensor system can be used in applications in which the overpassing of a temperature threshold level must be detected to prevent hazardous events such as a located fire in a corporate building, among others. To improve the use in wide range or real applications and hence, to improve their commercial feasibility, works to decrease significantly the optoelectronic costs are in progress.

\section{Acknowledgements}

This work has been supported by the Spanish TEC2010-20224-C02-02 project.

\section{References}

[1] Kashyap, Raman 'Fiber Bragg Gratings', Academic Press, ISBN: 0-12-400560-8, 1999.

[2] Cesar Jauregui, Tino Eidam, Jens Limpert, Andreas Tünnermann, "The impact of modal interference on the beam quality of highpower fiber amplifiers”. Optics Express, Volume: 19, Issue: 4, Pages: 3258-327, 2011.

[3] J. M. Lopez-Higuera. Handbook of optical fibre sensing technology, Wiley \& Sons (2002).

[4] Andrea Cusano, Antonello Cutolo and Jacques Albert (Eds)" Fiber Bragg Grating Sensors: Recent Advancements, Industrial Applications and Market Exploitation” Bentham Science Publishers Ltd, 2011.

[5] Manuel López-Amo1 and Jóse Miguel López-Higuera, “Multiplexing Techniques for FBG Sensors”, Chapter 6 of the book Fiber Bragg Grating Sensors, Bentham Science Publishers Ltd, 99-115, 2011.

[6] J.M. López-Higuera, L. Rodriguez-Cobo, A. Quintela, R. Hidalgo-Gato and H. Shokry Temperature level optical fiber sensor using shape memory alloy wires, OFS22, China, October, 2012. 